\title{
$\alpha$-isoptics of a triangle and their connection to $\alpha$-isoptic of an oval
}

\author{
Malgorzata Michalska (*) - Witold Mozgawa (**)
}

ABSTRACT - For a fixed positive angle $\alpha, \alpha<\pi$ we get an explicit formulas for an $\alpha$ isoptic curve of a triangle and study some of its properties. We use obtained results to show that $\alpha$-isoptic of an oval is an envelope of $\alpha$-isoptics of properly chosen triangles.

Mathematics Subject Classification (2010). 53A04; 52A10; 52A38.

KEYWORDS. Isoptic curve, convex curve, support function, envelope, oval.

\section{Introduction}

For two nontrivial vectors in the complex plane $u=u_{1}+i u_{2}$, $v=v_{1}+i v_{2}$ let $[u, v]=u_{1} v_{2}-u_{2} v_{1}$. On the other hand we know that $[u, v]=|u| \cdot|v| \sin \angle(u, v)$. Thus, we have the useful formula

$$
\sin \angle(u, v)=\frac{[u, v]}{|u| \cdot|v|} .
$$

An $\alpha$-isoptic curve $C_{\alpha}$ of a plane, closed, convex curve $C$ is a set of those points in the complex plane from which the curve $C$ is seen under a fixed angle $\pi-\alpha, \alpha \in(0, \pi)$. If $C$ is strictly convex and the origin of the plane is

(*) Indirizzo dell'A.: Institute of Mathematics, Maria Curie-Skłodowska University, pl. M. Curie-Sklodowska 1, 20-031 Lublin, Poland.

E-mail: malgorzata.michalska@poczta.umes.lublin.pl

(**) Indirizzo dell'A.: Institute of Mathematics, Maria Curie-Skłodowska University, pl. M. Curie-Skłodowska 1, 20-031 Lublin, Poland.

E-mail: mozgawa@poczta.umcs.lublin.pl 
chosen inside $C$, then there exists (cf. [2]) a differentiable function $p$ such that $p(t), t \in[0,2 \pi]$ is the distance from the origin to the support line. Function $p$ is called a support function and in its terms we have the parametrization of $C$

$$
z(t)=p(t) e^{i t}+\dot{p}(t) i e^{i t}, \quad t \in[0,2 \pi]
$$

and the parametrization of its $\alpha$-isoptic

$$
z_{\alpha}(t)=p(t) e^{i t}+\left\{-p(t) \cot \alpha+\frac{1}{\sin \alpha} p(t+\alpha)\right\} i e^{i t}, \quad t \in[0,2 \pi] .
$$

Properties of isoptics of strictly convex curves were studied in [4], [5], [9] and in [7] some results for not strictly convex curves can be found. Interesting extension of the notion of isoptic to non-euclidean spaces are given in [6].

In this paper we find an $\alpha$-isoptic curve of a triangle for a fixed positive angle $\alpha, \alpha<\pi$ and study some of its properties. As an application of our results we show that a family of $\alpha$-isoptics of properly chosen triangles has envelope which is $\alpha$-isoptic of an oval.

\section{Properties of an $\alpha$-isoptic curve of a triangle}

Let $\alpha$ be fixed and let $z_{k}=x_{k}+i y_{k}, k=1,2,3$, denote the vertices of a counter-clockwise oriented triangle $T$ on the complex plane C. If we ever use a subindex $k$ grater then 3 , we always mean it modulo 3 . For $k=1,2,3$ we use the following notations. Let $\overrightarrow{a_{k}}=\overrightarrow{z_{k+1} z_{k+2}}=$ $x_{k+2}-x_{k+1}+i\left(y_{k+2}-y_{k+1}\right)$ be an oriented side of the triangle $T$, then $a_{k}=\sqrt{\left(x_{k+2}-x_{k+1}\right)^{2}+\left(y_{k+2}-y_{k+1}\right)^{2}}$ denotes its length and $\beta_{k}$ is an angle of $T$ corresponding to the vertex $z_{k}$ and opposite to the side $\overrightarrow{a_{k}}$. Without loss of generality we can assume throughout this paper that $\beta_{1} \geq \beta_{2} \geq \beta_{3}$ or equivalently $a_{1} \geq a_{2} \geq a_{3}$.

From the inscribed angle theorem it is known that $C_{\alpha}$, the $\alpha$-isoptic curve of $T$ is a union of at least 3 and at most 6 circular arcs, one arc over each side of $T$ and if $\alpha$ is greater then $\pi-\beta_{k}$ one has an additional arc over the vertex $z_{k}$. We find the equations for each part of $C_{\alpha}$.

Over the side $\overrightarrow{a_{k}}$ a part of $C_{\alpha}$ is an arc of a circle $C\left(s_{k}, r_{k}\right)$ centered at the point $s_{k}$ and with radius $r_{k}$. Moreover, if $l_{k}$ is the line containing the side $\overrightarrow{a_{k}}$, then the center $s_{k}$ and the vertex $z_{k}$ lie in the same half plane of 
$l_{k}$ if and only if $\alpha$ is less then $\pi / 2$. To find the equation of the circle $C\left(s_{k}, r_{k}\right)$ we observe that the point $z=x+i y$ belongs to the part $C_{\alpha}$ over the side $\overrightarrow{a_{k}}$ if the angle between the vectors $\vec{u}=\overrightarrow{z z_{k+1}}$ and $\vec{v}=\overrightarrow{z z_{k+2}}$ is equal to $\pi-\alpha$. Using formula (1.1) we obtain the equation

$$
|u| \cdot|v| \sin (\pi-\alpha)=\left(x-x_{k+1}\right)\left(y-y_{k+2}\right)-\left(y-y_{k+1}\right)\left(x-x_{k+2}\right) .
$$

Taking square of both sides of the above equation we get after some calculations

$$
\left|z-\frac{\left(z_{k+1}+z_{k+2}\right) \pm i \cdot \cot \alpha \cdot\left(z_{k+1}-z_{k+2}\right)}{2}\right|^{2}=\frac{a_{k}^{2}}{4 \sin ^{2} \alpha} .
$$

Now, the property of the vertex $z_{k}$ and the center of the circle $s_{k}$ lying in the same half plane allow us to determine the sign in the above formula and finally we obtain

$$
r_{k}=\frac{a_{k}}{2 \sin \alpha},
$$

and

$$
s_{k}=\frac{\left(z_{k+1}+z_{k+2}\right)}{2}-i \cot \alpha \frac{\left(z_{k+1}-z_{k+2}\right)}{2} .
$$

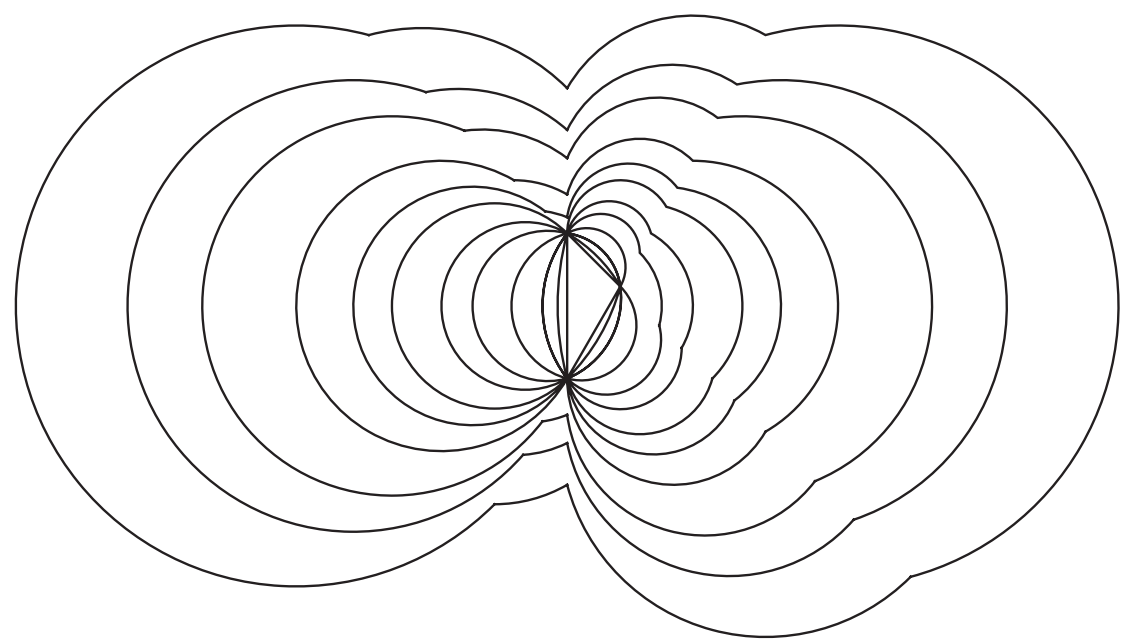

Figure 1. Isoptics of $T$ for $\alpha \in\{\pi / 12,5 \pi / 24,5 \pi / 12,7 \pi / 12,2 \pi / 3,3 \pi / 4$, $19 \pi / 24,5 \pi / 6,21 \pi / 24,43 \pi / 48,11 \pi / 12\}$. 
It is worth to notice that the arc of the circle $C\left(p_{k}, r_{k}\right)$ which is the part of $C_{\alpha}$ over the vertex $z_{k}$, for $\alpha$ less then $\pi-\beta_{k}$, is obtained in the same way. The only deference is that the center of the circle $p_{k}$ and vertex $z_{k}$ lie in the same half plane of $l_{k}$ if and only if $\alpha$ is greater then $\pi / 2$. Thus for $\alpha$ less then $\pi-\beta_{k}$ from (2.1) we have the following

$$
p_{k}=\frac{\left(z_{k+1}+z_{k+2}\right)}{2}+i \cot \alpha \frac{\left(z_{k+1}-z_{k+2}\right)}{2} .
$$

EXAMPLE 2.1. Let $T$ be a triangle with vertices $z_{1}=1, z_{2}=i$, $z_{3}=-i \sqrt{3}$. Then from formulas (2.1), (2.2), (2.3) and (2.4) we get the isoptics of $T$.

The most interesting question is for which $\alpha$ the $\alpha$-isoptic of $T$ is a convex curve. To study this problem we need to find the points of intersection of two circular arcs of $C_{\alpha}$.

If $\alpha$ is less or equal to $\pi-\beta_{k}$ then two circular arcs intersect at $z_{k}$. Let $\alpha$ be greater then $\pi-\beta_{k}$. Let $\zeta_{k}$ denote the intersection point of the circles $C\left(s_{k+1}, r_{k+1}\right), C\left(p_{k}, r_{k}\right)$ and the line $l_{k+2}$, and let $\eta_{k}$ denote the intersection point of the circles $C\left(s_{k+2}, r_{k+2}\right), C\left(p_{k}, r_{k}\right)$ and the line $l_{k+1}$. We can find the coordinates of $\zeta_{k}$ and $\eta_{k}$ by straightforward calculations. To simplify notations we put $\zeta_{k}=\eta_{k}=z_{k}$ for $\alpha \leq \pi-\beta_{k}$ and together we have

$$
\begin{aligned}
& \zeta_{k}=z_{k}+\left(z_{k+1}-z_{k}\right) \frac{a_{k+1} \cdot \min \left\{0, \sin \left(\alpha+\beta_{k}\right)\right\}}{a_{k+2} \sin \alpha}, \\
& \eta_{k}=z_{k}+\left(z_{k+2}-z_{k}\right) \frac{a_{k+2} \cdot \min \left\{0, \sin \left(\alpha+\beta_{k}\right)\right\}}{a_{k+1} \sin \alpha} .
\end{aligned}
$$

Now we state a useful property of $C_{\alpha}$ which helps us to study its convexity.

Proposition 2.2. Let $\alpha>\pi-\max \left\{\beta_{1}, \beta_{2}, \beta_{3}\right\}$ be fixed. Let $T$ be a given triangle in the complex plane and let $C_{\alpha}$ be its $\alpha$-isoptic curve. Then the triangle with the vertices $s_{k+1}, p_{k}, \zeta_{k}$ and the triangle with the vertices $p_{k}, s_{k+2}, \eta_{k}$ are geometrically congruent to each other and both are similar to $T$ for each $k \in\{1,2,3\}$ for which the arc of the circle $C\left(p_{k}, r_{k}\right)$ is a part of $C_{\alpha}$.

Proof. Let $k \in\{1,2,3\}$ be arbitrarily chosen. If $\alpha \leq \pi-\beta_{k}$ then none arc of the circle $C\left(p_{k}, r_{k}\right)$ is a part $C_{\alpha}$. Let $\alpha>\pi-\beta_{k}$. Then we 
have $\left|\overrightarrow{s_{k+1} \zeta_{k}}\right|=r_{k+1},\left|\overrightarrow{s_{k+2} \eta_{k}}\right|=r_{k+2}$ and $\left|\overrightarrow{p_{k} \zeta_{k}}\right|=\left|\overrightarrow{p_{k} \eta_{k}}\right|=r_{k}$. We can obtain lengths $\left|\overrightarrow{s_{k+2} p_{k}}\right|$ and $\left|\overrightarrow{s_{k+1} p_{k}}\right|$ using formulas (2.3) and (2.4), and we get the following equality

$$
\left|\overrightarrow{s_{k+2} p_{k}}\right|=\left|\frac{\left(z_{k+2}-z_{k}\right)}{2}+i \cot \alpha \frac{\left(z_{k+2}-z_{k}\right)}{2}\right|=r_{k+1},
$$

and analogously $\left|\overrightarrow{s_{k+1} p_{k}}\right|=r_{k+2}$. Thus the triangle with the vertices $s_{k+1}, p_{k}, \zeta_{k}$ and the triangle with the vertices $p_{k}, s_{k+2}, \eta_{k}$ have the required properties. Since $k$ was chosen arbitrarily we get the proof.

Proposition 2.2 allows us to reduce the domain of $\alpha$ to the interval $\left(0, \beta_{1}\right]$ while studying the convexity of $C_{\alpha}$. Namely we have

Corollary 2.3. Let $\alpha$ be fixed. If $\alpha>\pi-\max \left\{\beta_{1}, \beta_{2}, \beta_{3}\right\}$ then the $\alpha-$ isoptic curve of $T$ is not convex.

Proof. Due to our assumption $\beta_{1}=\max \left\{\beta_{1}, \beta_{2}, \beta_{3}\right\}$. Let $\alpha>\pi-\beta_{1}$ be fixed. Then, at least an arc of $C\left(p_{1}, r_{1}\right)$ is a part of $C_{\alpha}$. The vector $\overrightarrow{\zeta_{1} p_{1}}$ is normal to the tangent line to the circle $C\left(p_{1}, r_{1}\right)$ at the point $\zeta_{1}$ and the vector $\overrightarrow{\zeta_{1} s_{2}}$ is normal to the tangent line to the circle $C\left(s_{2}, r_{2}\right)$ at the point $\zeta_{1}$. By Proposition 2.2 and formula (1.1) we get that the circles $C\left(p_{1}, r_{1}\right)$ and $C\left(s_{2}, r_{2}\right)$ intersects at $\zeta_{1}$ under the angle $\pi-\beta_{3}$, thus $C_{\alpha}$ is not convex.

In fact, the domain of convexity of the curve $C_{\alpha}$ is a proper subset of $\left(0, \beta_{1}\right]$. We prove the following

THEOREM 2.4. Let $T$ be a given triangle in the complex plane and let $C_{\alpha}$ be its $\alpha$-isoptic curve. Then $C_{\alpha}$ is a convex curve if $\alpha \leq$ $\left(\pi-\max \left\{\beta_{1}, \beta_{2}, \beta_{3}\right\}\right) / 2$.

Proof. By our assumption we have $\beta_{1}=\max \left\{\beta_{1}, \beta_{2}, \beta_{3}\right\}$. Let $\alpha<\pi-\beta_{1}$ be fixed. Then $C_{\alpha}$ consists of 3 circular arcs $C\left(s_{k}, r_{k}\right), k=1,2,3$. Let $\gamma_{k}$ denote the angle under which the circles $C\left(s_{k+1}, r_{k+1}\right)$ and $C\left(s_{k+2}, r_{k+2}\right)$ intersect at the point $z_{k}$. Similarly as in the proof of Corollary 2.3 the vector $\overrightarrow{z_{k} s_{k+2}}$ is normal to the tangent line to the circle $C\left(s_{k+2}, r_{k+2}\right)$ at the point $z_{k}$ and the vector $\overrightarrow{z_{k} s_{k+1}}$ is normal to the tangent line to the circle $C\left(s_{k+1}, r_{k+1}\right)$ at the point $z_{k}$. Using formula (1.1) we find 
$\angle\left(\overrightarrow{z_{k} s_{k+2}}, \overrightarrow{z_{k} s_{k+1}}\right)=-2 \alpha-\beta_{k}$ and consequently $\gamma_{k}=\pi+2 \alpha+\beta_{k}$. Obviously $\gamma_{k} \leq 2 \pi$ and hence we get the required result.

COROLlary 2.5. The $\alpha$-isoptic curve of an equilateral polygon with $n$ sides is convex for $\alpha \leq \pi / n$ and the $(\pi / n)$-isoptic is a circle in which the polygon is inscribed.

Proof. If a polygon is an equilateral triangle then by Theorem 2.4 its $\alpha$ isoptic curve is convex for $\alpha \leq \pi / 3$ and $C_{\pi / 3}$ is a circle circumscribed on $T$.

Now let an equilateral polygon have $n$ sides, $n>3$. By inscribed angle theorem and Theorem 2.4 its $\alpha$-isoptic curve is convex for $\alpha \leq \pi / n$. And again the $(\pi / n)$-isoptic is a circle.

\section{The length of an $\alpha$-isoptic curve of a triangle}

In this section we study some properties of the length function $L(\alpha)$ of $C_{\alpha}$ as a function of $\alpha$. To find the length function we need some additional notations. For $k=1,2,3$ let $\varphi_{k}$ denote an angular measure in radians of the arc of the circle $C\left(s_{k}, r_{k}\right)$ which is a part of $C_{\alpha}$ and for a sufficiently large $\alpha$ let $\psi_{k}$ denote an angular measure in radians of the arc of the circle $C\left(p_{k}, r_{k}\right)$ which is also a part of $C_{\alpha}$. Then, by the inscribed angle theorem, we have for $k=1,2,3$

$$
\begin{aligned}
& \varphi_{k}=2\left(\alpha-\max \left\{0, \beta_{k+1}+\alpha-\pi\right\}-\max \left\{0, \beta_{k+2}+\alpha-\pi\right\}\right), \\
& \psi_{k}=2 \max \left\{0, \beta_{k}+\alpha-\pi\right\} .
\end{aligned}
$$

Using the above formulas we obtain the length of $C_{\alpha}$ as a function of $\alpha$

$$
L(\alpha)=\sum_{k=1}^{3}\left(\varphi_{k}+\psi_{k}\right) r_{k} .
$$

THEOREM 3.1. Let $T$ be a triangle in the complex plane and let $C_{\alpha}$ be an $\alpha$-isoptic curve of $T$ for a given angle $\alpha$. Then $L(\alpha)$ the length function of $C_{\alpha}$ defined by (3.3) is a continuous and strictly increasing function with respect to $\alpha$. The function $L$ is not convex.

Proof. Assume that $\beta_{1} \geq \beta_{2} \geq \beta_{3}$. Form (3.3) and the sine theorem we have the explicit formula for $L$ 
$L(\alpha)$

$$
\begin{aligned}
& = \begin{cases}\frac{2 \alpha R\left(\sin \beta_{1}+\sin \beta_{2}+\sin \beta_{3}\right)}{\sin \alpha} & \text { for } 0<\alpha \leq \pi-\beta_{1}, \\
\frac{2 R\left[2 \alpha \sin \beta_{1}+\left(\pi-\beta_{1}\right)\left(-\sin \beta_{1}+\sin \beta_{2}+\sin \beta_{3}\right)\right]}{\sin \alpha} & \text { for } \pi-\beta_{1}<\alpha \leq \pi-\beta_{2}, \\
\frac{2 R\left[\alpha\left(\sin \beta_{1}+\sin \beta_{2}-\sin \beta_{3}\right)+\left(\beta_{1}-\beta_{2}\right)\left(\sin \beta_{1}-\sin \beta_{2}\right)+\left(\pi+\beta_{3}\right) \sin \beta_{3}\right]}{\sin \alpha} & \text { for } \pi-\beta_{2}<\alpha \leq \pi-\beta_{3}, \\
\frac{4 R\left(\beta_{1} \sin \beta_{1}+\beta_{2} \sin \beta_{2}+\beta_{3} \sin \beta_{3}\right)}{\sin \alpha} & \text { for } \pi-\beta_{3}<\alpha<\pi,\end{cases} \\
& = \begin{cases}L_{1}(\alpha) & \text { for } 0<\alpha \leq \pi-\beta_{1}, \\
L_{2}(\alpha) & \text { for } \pi-\beta_{1}<\alpha \leq \pi-\beta_{2}, \\
L_{3}(\alpha) & \text { for } \pi-\beta_{2}<\alpha \leq \pi-\beta_{3}, \\
L_{4}(\alpha) & \text { for } \pi-\beta_{3}<\alpha<\pi,\end{cases}
\end{aligned}
$$

where $R$ is the radius of the circle circumscribing $T$. The straightforward calculations show that $L$ is continuous at the points $\pi-\beta_{1}, \pi-\beta_{2}, \pi-\beta_{3}$ and thus at all $\alpha \in(0, \pi)$. Each function $L_{k}(\alpha), k=1,2,3,4$ is differentiable in an open subset of its domain and the derivatives are equal to

$L_{1}^{\prime}(\alpha)=\frac{2 R}{\sin ^{2} \alpha}\left(\sin \beta_{1}+\sin \beta_{2}+\sin \beta_{3}\right)(\sin \alpha-\alpha \cos \alpha)$,

$L_{2}^{\prime}(\alpha)=\frac{2 R}{\sin ^{2} \alpha}\left[2 \sin \beta_{1}(\sin \alpha-\alpha \cos \alpha)-\left(\pi-\beta_{1}\right)\left(-\sin \beta_{1}+\sin \beta_{2}+\sin \beta_{3}\right) \cos \alpha\right]$,

$L_{3}^{\prime}(\alpha)=\frac{2 R}{\sin ^{2} \alpha}\left\{\left(\sin \beta_{1}+\sin \beta_{2}-\sin \beta_{3}\right)(\sin \alpha-\alpha \cos \alpha)\right.$

$\left.-\left[\left(\beta_{1}-\beta_{2}\right)\left(\sin \beta_{1}-\sin \beta_{2}\right)+\left(\pi+\beta_{3}\right) \sin \beta_{3}\right] \cos \alpha\right\}$,

$L_{4}^{\prime}(\alpha)=\frac{-4 R}{\sin ^{2} \alpha}\left(\beta_{1} \sin \beta_{1}+\beta_{2} \sin \beta_{2}+\beta_{3} \sin \beta_{3}\right) \cos \alpha$.

Since they have property

$$
L_{k+1}^{\prime}\left(\pi-\beta_{k}\right)-L_{k}^{\prime}\left(\pi-\beta_{k}\right)=\frac{a_{k}-a_{k+1}-a_{k+2}}{\sin \beta_{k}}<0, \quad \text { for } k=1,2,3,
$$

thus the function $L^{\prime}$ is not defined at the points $\pi-\beta_{1}, \pi-\beta_{2}, \pi-\beta_{3}$.

We show that $L^{\prime}$ is a positive function in each interval of its domain and thus $L$ is increasing.

First note that the function $f(\alpha)=\sin \alpha-\alpha \cos \alpha$ is positive and increasing for $\alpha \in(0, \pi)$ and the function $g(\alpha)=-\cos \alpha$ is positive for $\alpha \in(\pi / 2, \pi)$. Consequently the functions $L_{1}^{\prime}, L_{3}^{\prime}, L_{4}^{\prime}$ are positive in their domains. 
The function $L_{2}^{\prime}$ is positive for $\alpha>\pi / 2$ hence we have to show that it is positive in the interval $\left[\pi-\beta_{1}, \pi / 2\right]$. Since the function

$$
h(\alpha)=2 \sin \beta_{1}(\sin \alpha-\alpha \cos \alpha)-\left(\pi-\beta_{1}\right)\left(-\sin \beta_{1}+\sin \beta_{2}+\sin \beta_{3}\right) \cos \alpha
$$

has nonnegative derivative for $\alpha \in\left[\pi-\beta_{1}, \pi / 2\right]$ it is enough to show that $h\left(\pi-\beta_{1}\right)$ is positive. Let

$$
\begin{aligned}
& H\left(\beta_{2}, \beta_{3}\right)=h\left(\pi-\beta_{1}\right) \\
& =2 \sin ^{2}\left(\beta_{2}+\beta_{3}\right)-\left(\beta_{2}+\beta_{3}\right) \cos \left(\beta_{2}+\beta_{3}\right)\left[\sin \left(\beta_{2}+\beta_{3}\right)+\sin \beta_{2}+\sin \beta_{3}\right] .
\end{aligned}
$$

We need to show that the minimal value of $H$ in

$$
D=\left\{\left(\beta_{2}, \beta_{3}\right) \mid 0 \leq \beta_{3} \leq \beta_{2} \leq \pi / 2,0 \leq \beta_{2}+\beta_{3} \leq \pi / 2\right\}
$$

is nonnegative. The function $H$ has no critical points inside $D$. Moreover,

$$
\begin{aligned}
& H\left(\beta_{2}, \pi / 2-\beta_{2}\right)=2, \quad \text { for } \beta_{2} \in[\pi / 4, \pi / 2], \\
& H\left(\beta_{2}, 0\right)=2 \sin \beta_{2}\left(\sin \beta_{2}-\beta_{2} \cos \beta_{2}\right) \geq 0, \text { for } \beta_{2} \in[0, \pi / 2] .
\end{aligned}
$$

To complete this part of the proof we show that

$$
\widetilde{H}\left(\beta_{2}\right)=H\left(\beta_{2}, \beta_{2}\right)=2 \sin ^{2} 2-2 \beta_{2} \cos 2 \beta_{2}\left(\sin 2 \beta_{2}-2 \sin \beta_{2}\right)
$$

is nonnegative for $\beta_{2} \in[0, \pi / 4]$. Once again, we use the fact that this function is nondecreasing and $\widetilde{H}(0)=0$. Indeed, we have

$$
\begin{aligned}
& \widetilde{H}^{\prime}\left(\beta_{2}\right)=8 \cos \frac{\beta_{2}}{2}\left[\left(3 \cos \beta_{2}-1\right) \sin \frac{\beta_{2}}{2} \cos 2 \beta_{2}+\beta_{2}\left(\cos 2 \beta_{2}-2 \cos 3 \beta_{2}\right) \cos \frac{\beta_{2}}{2}\right] \\
& =8 \cos \frac{\beta_{2}}{2}\left\{\left[\cos \frac{5 \beta_{2}}{2} \sin ^{2} \frac{\beta_{2}}{2}\left(\beta_{2}-\sin \beta_{2}\right)\right]+\left[\sin \frac{5 \beta_{2}}{2}\left(3 \cos \beta_{2}-1\right) \sin ^{2} \frac{\beta_{2}}{2}\right]\right. \\
& \left.+\left[\cos \frac{5 \beta_{2}}{2}\left(\sin \beta_{2} \cos \beta_{2}-\beta_{2}\right)+\frac{3}{2} \beta_{2} \sin \beta_{2} \sin \frac{5 \beta_{2}}{2}\right]\right\} .
\end{aligned}
$$

From the first formula we obtain that $\widetilde{H}^{\prime}\left(\beta_{2}\right)$ is positive for $\beta_{2} \in$ $(\pi / 6, \pi / 4]$. If $\beta_{2} \in(0, \pi / 6]$ each term in a square bracket in the second formula is positive. Thus, $\widetilde{H}^{\prime}\left(\beta_{2}\right)$ is positive for $\beta_{2} \in(0, \pi / 4]$. Finally, we get that $H\left(\beta_{2}, \beta_{3}\right)$ is nonnegative and is equal to 0 only if $\beta_{2}=\beta_{3}=0$. This completes the proof that $L_{2}^{\prime}$ is positive in its domain.

Moreover, the functions $L_{k}^{\prime}, k=1,2,3,4$ are positive and the property (3.4) implies that the graph of $L$ is not convex. It is worth to notice that each $L_{k}, k=1,2,3,4$ is convex in its domain since $\left(f(\alpha) / \sin ^{2} \alpha\right)^{\prime}=$ $\alpha\left(1+\cos ^{2} \alpha\right) / \sin ^{3} \alpha>0$ and $\left(-g(\alpha) / \sin ^{2} \alpha\right)^{\prime}=\left(1+\cos ^{2} \alpha\right) / \sin ^{3} \alpha>0$ for $\alpha \in(0, \pi)$. 
ExAmple 3.2. For the triangle $T$ from Example 2.1 the length function $L(\alpha)$ given by (3.3) has the graph shown on Figure 2 .

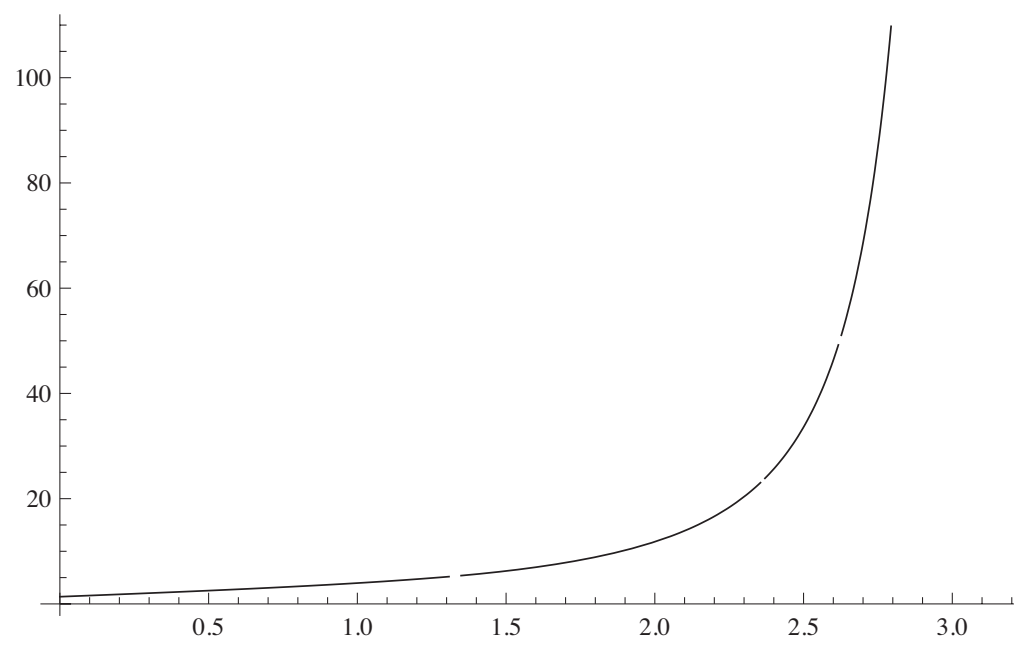

Figure 2. Graph of the length function $L(\alpha)$ of $T$.

\section{The area of an $a$-isoptic curve of a triangle}

Our aim in this section is to investigate some properties of the area function $A(\alpha)$ of the $\alpha$-isoptic curve of $T$ as a function of $\alpha$. Let $\zeta_{1} \eta_{1} \zeta_{2} \eta_{2}, \zeta_{3} \eta_{3}$, where $\zeta_{k}$ and $\eta_{k}$ are defined by (2.5) and (2.6), respectively, be a counterclockwise oriented polygon. If $\eta_{k}=\zeta_{k}=z_{k}$ then the point $z_{k}$ is counted only once in the polygon. Using formulas (3.1) and (3.2) we get the area function of $C_{\alpha}$

$$
\begin{aligned}
& A(\alpha)=\text { area of } \zeta_{1} \eta_{1} \zeta_{2} \eta_{2} \zeta_{3} \eta_{3} \\
& +\sum_{k=1}^{3}\left(\varphi_{k} r_{k}^{2}-\frac{1}{2} r_{k}^{2} \sin \varphi_{k}\right)+\sum_{k=1}^{3}\left(\psi_{k} r_{k}^{2}-\frac{1}{2} r_{k}^{2} \sin \psi_{k}\right) .
\end{aligned}
$$

The behavior of the function $A(\alpha)$ is described in the following

THeOREm 4.1. Let $T$ be a triangle in the complex plane and let $C_{\alpha}$ be an $\alpha$-isoptic curve of $T$ for a given angle $\alpha$. Then $A(\alpha)$, the area function of $C_{\alpha}$ defined by (4.1) is a differentiable, strictly increasing and convex function with respect to $\alpha$. 
Proof. Assume that $\beta 1 \geq \beta_{2} \geq \beta_{3}$ and let $\zeta_{k}$ and $\eta_{k}$ be defined by (2.5) and (2.6), respectively. To compute the area function given by (4.1) we need to find the area of a polygon $\zeta_{1} \eta_{1} \zeta_{2} \eta_{2} \zeta_{3} \eta_{3}$. To this end we use theorem 3 given by Radić in [10]. He proved that

$$
\text { area of } \zeta_{1} \eta_{1} \zeta_{2} \eta_{2} \zeta_{3} \eta_{3}=\frac{1}{2}\left|\zeta_{1}+\eta_{1}, \eta_{1}+\zeta_{2}, \zeta_{2}+\eta_{2}, \eta_{2}+\zeta_{3}, \zeta_{3}+\eta_{3}, \eta_{3}+\zeta_{1}\right| \text {, }
$$

where

$$
\left|z_{1}+z_{2}, z_{2}+z_{3}, \ldots, z_{n}+z_{1}\right|=\sum_{1 \leq i<j \leq n}(-1)^{3+i+j}\left[z_{i}, z_{j}\right] .
$$

Obviously, if $\eta_{k}=\zeta_{k}=z_{k}$ then the point $z_{k}$ is counted only once in the polygon. Applying the sine theorem we get $A(\alpha)$

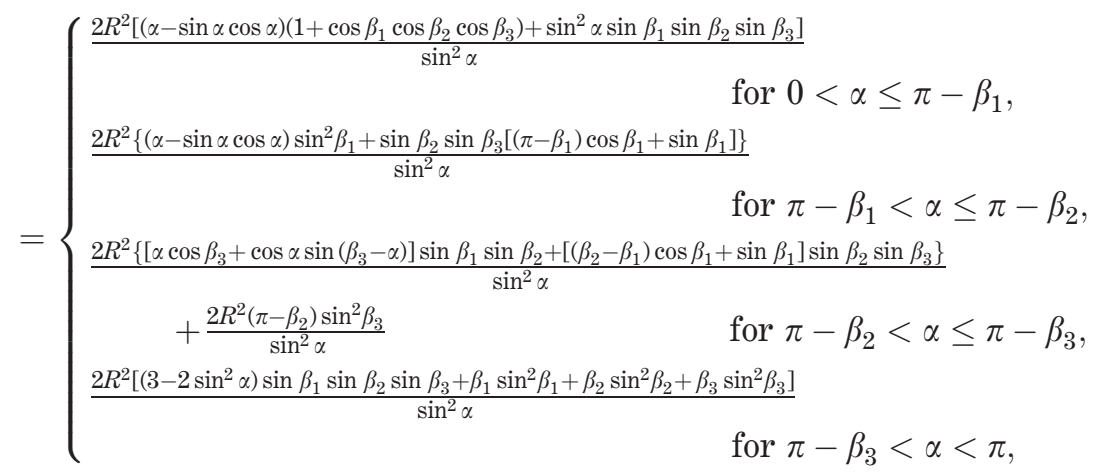$$
= \begin{cases}A_{1}(\alpha) & \text { for } 0<\alpha \leq \pi-\beta_{1}, \\ A_{2}(\alpha) & \text { for } \pi-\beta_{1}<\alpha \leq \pi-\beta_{2}, \\ A_{3}(\alpha) & \text { for } \pi-\beta_{2}<\alpha \leq \pi-\beta_{3}, \\ A_{4}(\alpha) & \text { for } \pi-\beta_{3}<\alpha<\pi,\end{cases}
$$

where $R$ is the radius of the circle circumscribed on $T$. The function $A(\alpha)$ is continuous at the points $\pi-\beta_{1}, \pi-\beta_{2}, \pi-\beta_{3}$ and thus at all $\alpha \in(0, \pi)$. The same is true for its derivative and we have

$$
\begin{aligned}
& A_{1}^{\prime}(\alpha)=\frac{4 R^{2}}{\sin ^{3} \alpha}(\sin \alpha-\alpha \cos \alpha)\left(1+\cos \beta_{1} \cos \beta_{2} \cos \beta_{3}\right), \\
& A_{2}^{\prime}(\alpha)=\frac{4 R^{2}}{\sin ^{3} \alpha}\left\{(\sin \alpha-\alpha \cos \alpha) \sin ^{2} \beta_{1}\right. \\
& \left.-\cos \alpha \sin \beta_{2} \sin \beta_{3}\left[\left(\pi-\beta_{1}\right) \cos \beta_{1}+\sin \beta_{1}\right]\right\},
\end{aligned}
$$




$$
\begin{aligned}
& A_{3}^{\prime}(\alpha)=\frac{4 R^{2}}{\sin ^{3} \alpha}\left\{\left[\sin \left(\alpha-\beta_{3}\right)-\alpha \cos \alpha \cos \beta_{3}\right] \sin \beta_{1} \sin \beta_{2}\right. \\
& \left.\quad-\cos \alpha\left[\left(\pi-\beta_{2}\right) \sin \beta_{1} \cos \beta_{2} \sin \beta_{3}+\left[\left(\pi-\beta_{1}\right) \cos \beta_{1}+\sin \beta_{1}\right] \sin \beta_{2} \sin \beta_{3}\right]\right\},
\end{aligned}
$$

$A_{4}^{\prime}(\alpha)=\frac{-4 R^{2}}{\sin ^{3} \alpha} \cos \alpha\left[3 \sin \beta_{1} \sin \beta_{2} \sin \beta_{3}+\beta_{1} \sin ^{2} \beta_{1}+\beta_{2} \sin ^{2} \beta_{2}+\beta_{3} \sin ^{2} \beta_{3}\right]$.

where the derivative of the function $A_{k}(\alpha), k=1,2,3,4$, is defined in its domain. Using functions $f$ and $g$ defined in the proof of Theorem 3.1 we immediately obtain that $A_{1}^{\prime}, A_{3}^{\prime}, A_{4}^{\prime}$ are positive in their domains. Moreover, since $f(\alpha) \geq f\left(\pi-\beta_{1}\right)$ we have

$A_{2}^{\prime}(\alpha) \geq \frac{2 f(\alpha)\left(\sin ^{2} \beta_{1}-\cos \alpha \sin \beta_{2} \sin \beta_{3}\right)}{\sin ^{3} \alpha} \geq \frac{2 f(\alpha)\left(\sin ^{2} \beta_{1}-\sin \beta_{2} \sin \beta_{3}\right)}{\sin ^{3} \alpha}>0$.

Since $A^{\prime}(\alpha)$ is positive then the function $A(\alpha)$ is strictly increasing.

The second derivative of the function $A_{k}(\alpha), k=1,2,3,4$, is defined in each open subset of its domain and it is equal to

$$
\begin{aligned}
& A_{1}^{\prime \prime}(\alpha)=\frac{4 R^{2}}{\sin ^{4} \alpha}\left[\alpha\left(1+2 \cos ^{2} \alpha\right)-3 \sin \alpha \cos \alpha\right]\left(1+\cos \beta_{1} \cos \beta_{2} \cos \beta_{3}\right), \\
& A_{2}^{\prime \prime}(\alpha)=\frac{4 R^{2}}{\sin ^{4} \alpha}\left\{\left[\alpha\left(1+2 \cos ^{2} \alpha\right)-3 \sin \alpha \cos \alpha\right] \sin ^{2} \beta_{1}\right. \\
& \left.+\left(1+2 \cos ^{2} \alpha\right)\left[\left(\pi-\beta_{1}\right) \cos \beta_{1}+\sin \beta_{1}\right] \sin \beta_{2} \sin \beta_{3}\right\} \\
& A_{3}^{\prime \prime}(\alpha)=\frac{4 R^{2}}{\sin ^{4} \alpha}\left\{\left[\alpha\left(1+2 \cos ^{2} \alpha\right)-3 \sin \alpha \cos \alpha\right] \sin \beta_{1} \sin \beta_{2} \cos \beta_{3}\right. \\
& +\left(1+2 \cos ^{2} \alpha\right)\left[\left(\pi-\beta_{2}\right) \sin \beta_{1} \cos \beta_{2} \sin \beta_{3}\right. \\
& \left.\left.+\left(\left(\pi-\beta_{1}\right) \cos \beta_{1}+\sin \beta_{1}\right) \sin \beta_{2} \sin \beta_{3}+\sin \beta_{1} \sin \beta_{2} \sin \beta_{3}\right]\right\} \\
& A_{4}^{\prime \prime}(\alpha)=\frac{4 R^{2}}{\sin ^{4} \alpha}\left(1+2 \cos { }^{2} \alpha\right)\left[3 \sin \beta_{1} \sin \beta_{2} \sin \beta_{3}+\beta_{1} \sin ^{2} \beta_{1}\right. \\
& \left.+\beta_{2} \sin ^{2} \beta_{2}+\beta_{3} \sin ^{2} \beta_{3}\right] .
\end{aligned}
$$


Thus the function $A^{\prime \prime}(\alpha)$ is not continuous at the points $\pi-\beta_{1}, \pi-\beta_{2}$, $\pi-\beta_{3}$, but still, it is positive in its domain. This completes the proof.

ExAmple 4.2. Once again, let us take the triangle $T$ from Example 2.1. Then the graph of the area function $A(\alpha)$ given by (4.1) is shown on Figure 3.

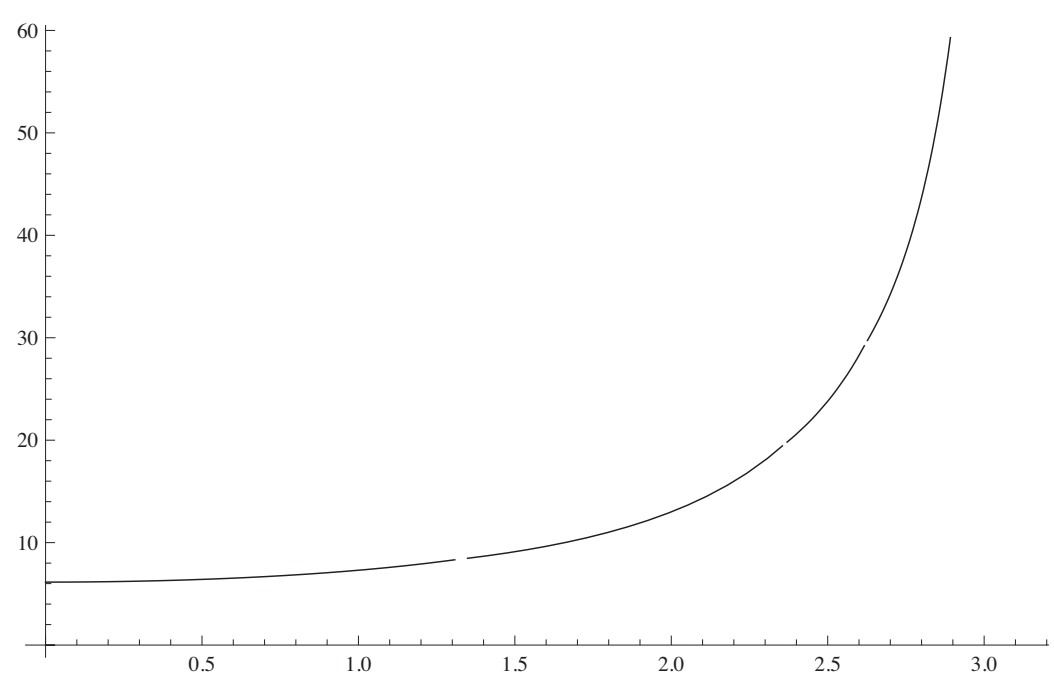

Figure 3. Graph of the area function $A(\alpha)$ of $T$.

\section{Application of $\alpha$-isoptic curves of a triangle}

Let $\alpha \in(0, \pi)$ be fixed. In this section we use results obtained in Section 2 to study the $\alpha$-isoptic $C_{\alpha}$ of an oval $C$. By an oval we understand $C^{2}$, plane closed simple curve with positive curvature.

Let $p(t) \in C^{2}([0,2 \pi]), t \in[0,2 \pi]$ be the support function of $C$. Then the point $z_{\alpha}(t)$ satisfying (1.3) belongs to $C_{\alpha}$ and it is an intersection of two lines tangent to $C$ at points $z(t)$ and $z(t+\alpha)$. Let $\xi$ be an arbitrary point from an open angle $\angle\left(\overrightarrow{z_{\alpha}(t) z(t+\alpha)}, \overrightarrow{z_{\alpha}(t) z(t)}\right)$ and let $T$ be a counter-clockwise oriented triangle with vertices $z(t+\alpha), z(t), \xi$. By $C_{\alpha, t}$ we denote an arc of a circle

$$
\left|z-\frac{(z(t+\alpha)+z(t))+i \cot \alpha(z(t+\alpha)-z(t))}{2}\right|=\frac{|z(t+\alpha)-z(t)|}{2 \sin \alpha}
$$

which is also a part of $\alpha$-isoptic of $T$. We should mention that $C_{\alpha, t}$ does not depend on $\xi$. If $[\overrightarrow{\xi z(t+\alpha)}, \overrightarrow{\xi z(t)}]>0$ then the center of the circle in (5.1) is 
obtained from equation (2.4), overwise it is obtained from equation (2.3). Finally we define the family of arcs as follows

$$
\mathcal{F}_{\alpha}=\left\{C_{\alpha, t}, t \in[0,2 \pi]\right\} .
$$

Using the above notations we have

THEOREM 5.1. $\quad C_{\alpha}$ is the envelope of the family $\mathcal{F}_{\alpha}$ defined by (5.2).

Proof. Let $F(x, y, t)=0$ denote an equation for the family $\mathcal{F}_{\alpha}$ given by (5.2). Then, applying formula (1.2) to (5.1) with $z=x+i y$, we get

$$
\begin{aligned}
& F(x, y, t)=\left(x^{2}+y^{2}\right) \sin \alpha \\
& +x[p(t+\alpha) \sin t+\dot{p}(t+\alpha) \cos t-p(t) \sin (t+\alpha)-\dot{p}(t) \cos (t+\alpha)] \\
& +y[-p(t+\alpha) \cos t+\dot{p}(t+\alpha) \sin t+p(t) \cos (t+\alpha)-\dot{p}(t) \sin (t+\alpha)] \\
& +p(t+\alpha) \dot{p}(t)-p(t) \dot{p}(t+\alpha)=0,
\end{aligned}
$$

thus $\mathcal{F}_{\alpha}$ is indeed a one parameter family of arcs. Moreover, the point

$$
\begin{aligned}
& z_{\alpha}(t)=x_{\alpha}(t)+i y_{\alpha}(t) \\
& =\frac{p(t) \sin (t+\alpha)-p(t+\alpha) \sin t+i(-p(t) \cos (t+\alpha)+p(t+\alpha) \cos t)}{\sin \alpha}
\end{aligned}
$$

defined by (1.3) satisfies the equation (5.3) and thus $z_{\alpha}(t) \in C_{\alpha, t}$ since $\xi$ is an interior point of the angle $\angle\left(\overrightarrow{z_{\alpha}(t) z(t+\alpha)}, \overrightarrow{z_{\alpha}(t) z(t)}\right)$.

Now Theorem 4 in [1] asserts that $C_{\alpha, t}$ and $C_{\alpha}$ are tangent at the point $z_{\alpha}(t)$. To show that $C_{\alpha}$ is the envelope of $\mathcal{F}_{\alpha}$ it is enough to check that $F_{t}^{\prime}(x, y, t)=0$ at $z_{\alpha}(t)$ (see, e.g., [3] or [11]). Indeed, we have

$$
\begin{aligned}
& F_{t}^{\prime}(x, y, t)=x[R(t+\alpha) \cos t-R(t) \cos (t+\alpha)] \\
& +y[R(t+\alpha) \sin t+R(t) \sin (t+\alpha)]+p(t+\alpha) R(t)-p(t) R(t+\alpha),
\end{aligned}
$$

where $R(t)=p(t)+\ddot{p}(t)$ is a radius of curvature of $C$, and finally,

$$
F_{t}^{\prime}\left(x_{\alpha}(t), y_{\alpha}(t), t\right)=0
$$

which completes the proof.

Theorem 5.1 remains true in special case when $T$ is inscribed in oval $C$.

The above considerations can be related to those in paper of Martini [8] on the classical light field theory in $\mathbb{R}^{d}, d \geq 2$. 


\section{REFERENCES}

[1] K. Benko - W. Cieślak - S. Góźdź - W. Mozgawa, On isoptic curves, An. Științ. Univ. Al. I. Cuza Iași Secț. I a Mat., 36 (1990), no. 1, 47-54.

[2] T. Bonnesen - W. Fenchel, Theorie der konvexen Körper, Chelsea Publ. Comp., New York, 1948.

[3] J. W. Bruce - P. J. Giblin, Curves and singularities. A geometrical introduction to singularity theory, Cambridge University Press, Cambridge, 1984.

[4] W. Cieślak - A. Miernowski - W. Mozgawa, Isoptics of a closed strictly convex curve, Lect. Notes in Math., 1481 (1991), 28-25.

[5] W. Cieślak - A. Miernowski - W. Mozgawa, Isoptics of a closed strictly convex curve. II, Rend. Sem. Mat. Univ. Padova, 96 (1996), 37-49.

[6] G. Csima - J. SzIRMaI, Isoptic curves of conic sections in constant curvature geometries, to appear.

[7] Ákurusa, Is a convex plane body determined by an isoptic?, Beitr. Algebra Geom., 53 (2012), 281-294.

[8] H. Martini, A contribution to the light field theory, Beitr. Algebra Geom., 30 (1990), 193-201.

[9] M. Michalska, A sufficient condition for the convexity of the area of an isoptic curve of an oval, Rend. Sem. Mat. Univ. Padova, 110 (2003), 161-169.

[10] M. Radić, About a Determinant of Rectangular $2 \times n$ Matrix and its Geometric Interpretation, Beitr. Algebra Geom., 46 (2005), No. 1, 321-349.

[11] E. W. Weisstein “Envelope.” From MathWorld - A Wolfram Web Resource. http://mathworld.wolfram.com/Envelope.html.

Manoscritto pervenuto in redazione il 4 novembre 2013. 